\title{
Leptospira species and serovars identified by MALDI-TOF mass spectrometry after database implementation
}

\author{
Adriana Calderaro*, Giovanna Piccolo, Chiara Gorrini, Sara Montecchini, Mirko Buttrini, Sabina Rossi, \\ Maddalena Piergianni, Flora De Conto, Maria Cristina Arcangeletti, Carlo Chezzi and Maria Cristina Medici
}

\begin{abstract}
Background: Leptospirosis, a spirochaetal zoonotic disease of worldwide distribution, endemic in Europe, has been recognized as an important emerging infectious disease, though yet it is mostly a neglected disease which imparts its greatest burden on impoverished populations from developing countries. Leptospirosis is caused by the infection with any of the more than 230 serovars of pathogenic Leptospira sp. In this study we aimed to implement the MALDI-TOF mass spectrometry (MS) database currently available in our laboratory with Leptospira reference pathogenic (L. interrogans, L. borgpetersenii, L. kirschneri, L. noguchii), intermediate (L. fainei) and saprophytic (L. biflexa) strains of our collection in order to evaluate its possible application to the diagnosis of leptospirosis and to the typing of strains. This was done with the goal of understanding whether this methodology could be used as a tool for the identification of Leptospira strains, not only at species level for diagnostic purposes, but also at serovar level for epidemiological purposes, overcoming the limits of serological and molecular conventional methods. Twenty Leptospira reference strains were analysed by MALDI-TOF MS. Statistical analysis of the protein spectra was performed by ClinProTools software.

Results: The spectra obtained by the analysis of the reference strains tested were grouped into 6 main classes corresponding to the species analysed, highlighting species-specific protein profiles. Moreover, the statistical analysis of the spectra identified discriminatory peaks to recognize Leptospira strains also at serovar level extending previously published data.
\end{abstract}

Conclusions: In conclusion, we confirmed that MALDI-TOF MS could be a powerful tool for research and diagnostic in the field of leptospirosis with broad applications ranging from the detection and identification of pathogenic leptospires for diagnostic purposes to the typing of pathogenic and non-pathogenic leptospires for epidemiological purposes in order to enrich our knowledge about the epidemiology of the infection in different areas and generate control strategies.

Keywords: Leptospira sp., MALDI-TOF MS, Identification, Database implementation

\section{Background}

Leptospirosis, a spirochaetal zoonotic disease of worldwide distribution [1,2], endemic in Europe, has been recognized as an important emerging infectious disease [3], though yet it is mostly a neglected disease which imparts its greatest burden on impoverished populations from developing countries [2]. More than 1 million cases of severe leptospirosis occur worldwide annually [4] and the disease varies from a

\footnotetext{
* Correspondence: adriana.calderaro@unipr.it

Unit of Microbiology and Virology, Department of Clinical and Experimental Medicine, University of Parma, Viale A. Gramsci, 14-43126 Parma, Italy
}

sub clinical infection to a severe illness with multi-organ involvement, leading to a fatal form in some cases. Leptospirosis is caused by the infection with any of the more than 230 serovars of pathogenic Leptospira sp. [4], clustered into 24 serogroups as a function of antigenic determinants and into 20 genomospecies, on the basis of genetic methods $[3,5,6]$. Evidence was demonstrated that serogrouping does not strictly equate with speciation, because some serovars within the same serogroup may be distributed among different species $[5,6]$. 
The identification of the causative Leptospira isolate at the species level allows to determine its pathogenic status, the probable source of infection and to distinguish sporadic cases from outbreaks [7]. The gold standard methodology for leptospirosis diagnosis, the microscopic agglutination test (M.A.T.), is based on the antibody response of the host, which can occur only in a period of 8-10 days after the onset of symptoms (seroconversion) [8], and it presents the inconvenience of being laborious and requires the maintenance of living cultures. Serotyping requires an indaginous technical procedure providing an ambiguous identification of Leptospira isolates and it is performed only in reference laboratories [7]. Moreover, several molecular assays till now proposed [5,9-13] cannot be routinely applied to the identification of Leptospira isolates since they do not allow for unambiguous identification of Leptospira isolates, needing for sequencing-based techniques [6,7,14-19].

Matrix-assisted laser desorption ionization-time-of-flight mass spectrometry (MALDI-TOF MS) emerged over the last years as a first line technique for the rapid bacterial identification [20,21]. Recently, in a study by our research group [22], MALDI-TOF MS was applied for the first time on Brachyspira strains of human and animal origins, overcoming the problems previously encountered in the identification of these spirochaetes when using biochemical and genetic-based methods. Furthermore, we also applied this technology to the identification of Borrelia sp. strains at species level [23].

In the field of leptospirosis, Djelouadji et al. [7] and Rettinger et al. [24] evaluated the application of MALDITOF MS for the identification of Leptospira sp. strains. In this study, performed in our Regional Reference Laboratory for Leptospirosis of the Italian Ministry of Health we analysed our own panel of Leptospira reference pathogenic (L. interrogans, L. borgpetersenii, L. kirschneri, L. noguchii), intermediate (L. fainei) and saprophytic (L. biflexa) strains circulating in Italy with the aim to supplement our MALDI-TOF database. In this way, the possible application of the MALDI-TOF MS to the diagnosis of leptospirosis and to the typing of strains was evaluated including both Leptospira strains already tested at species level by previous published papers $[7,24]$ and Leptospira strains not investigated before. This was done with the intent to make a further contribution to understand whether this methodology could be used as a tool for the identification of Leptospira strains, not only at species level for diagnostic purposes, but also at serovar level for epidemiological purposes, overcoming the limits of serological and molecular conventional methods.

\section{Methods}

Leptospira strains

A total of 20 Leptospira reference strains, 18 provided for diagnostic purposes (these strains are also authorized to be utilized for research use) from the Istituto Superiore di Sanità, Rome, Italy, National Reference Laboratory for Leptospirosis (L. interrogans, L. borgpetersenii, L. kirschneri and L. biflexa) and from the Centre National des Leptospires, Institut Pasteur, Paris (L. noguchii and L. fainei), were included in this study (Table 1 ).

These strains were cultured on Ellinghausen-McCullough Johnson and Harris (EMJH) medium (Difco ${ }^{\mathrm{TM}}$ Leptospira Medium Base EMJH and Difco ${ }^{\mathrm{TM}}$ Leptospira Enrichment EMJH BD, USA) at $30^{\circ} \mathrm{C}$ [8]. Two different lots of this medium (lots no. 1311945 and no. 2299369) were tested in order to ensure the reproducibility of protein profiles. Microscopic observation was performed to exclude the presence of microorganisms other than leptospires and to appreciate the Leptospira sp. growth in cultures that were periodically sub-cultured into fresh media. Bacteria used for MALDI-TOF MS measurements and for the creation of protein reference spectra were cultured for 7 days.

The strains belonging to the pathogenic species L. interrogans, L. borgpetersenii and L. kirschneri are currently used to perform the M.A.T. in our laboratory that is the Regional Reference Laboratory for Leptospirosis of the Italian Ministry of Health.

\section{MALDI-TOF MS analysis}

After 7 days of growth, bacteria were counted by microscopy and an aliquot of $1 \mathrm{ml}$ containing at least $1 \times 10^{6}$ leptospires per $\mathrm{ml}$ was utilized for ethanol/formic acid extraction, as previously described [22]. The obtained extracts were spotted 40 times onto a "MSP-96 polished steel" target plate (Bruker Daltonics, Germany). Immediately after drying, all spots were overlaid with $1 \mu \mathrm{l}$ of matrix, a saturated solution of $\alpha$-cyano-4-hydroxycinnamic acid (HCCA) (Bruker Daltonics, Germany) dissolved in $50 \%$ CAN and $2.5 \%$ trifluoroacetic acid (TFA) (SigmaAldrich, Milan, Italy).

In each plate Escherichia coli (ATCC 8739) was used as a positive control and a non-inoculated matrix solution was used as a negative control. Each spot was measured in 40-shot steps for a total of 240 laser shots. MALDI-TOF MS measurements were performed by a Microflex LT mass spectrometer (Bruker Daltonics, Germany) provided with a $20 \mathrm{~Hz}$ nitrogen laser using FlexControl software (version 3.3.63, Bruker Daltonics, Germany). Spectra were obtained in the linear positive mode with an accelerating voltage of $20 \mathrm{kV}$ and analysed within a mass range of 2,000-20,000 Da. Pre-processing and identification steps were performed using the manufacturer's parameters. Before each measurement, the instrument was calibrated using the Bacterial Test Standard (BTS) provided by Bruker Daltonics. Preparation of the BTS and calibration were performed following the manufacturer's instructions: calibration was successful when proteins of the mass spectra were in a range of \pm 300 parts per million (ppm). 
Table 1 Twenty Leptospira reference serovars belonging to 6 species used for MALDI-TOF MS analysis

\begin{tabular}{|c|c|c|c|c|c|}
\hline No. & Serovar & Serogroup & Strain & Genomospecies & Pathogenicity \\
\hline 1 & Autumnalis & Autumnalis & Akiyami A & L. interrogans & Pathogenic \\
\hline 2 & Bataviae & Bataviae & Pavia 1 & L. interrogans & Pathogenic \\
\hline 3 & Bratislava & Australis & Riccio2 & L. interrogans & Pathogenic \\
\hline 4 & Canicola & Canicola & Alarik & L. interrogans & Pathogenic \\
\hline 5 & Copenhageni & Icterohaemorrhagiae & Wijnberg & L. interrogans & Pathogenic \\
\hline 6 & Hardjo & Sejroe & Hardjoprajitno & L. interrogans & Pathogenic \\
\hline 7 & Hardjo & Sejroe & Farina C. 715 & L. interrogans & Pathogenic \\
\hline 8 & Hebdomadis & Hebdomadis & Hebdomadis $\mathrm{H}$ & L. interrogans & Pathogenic \\
\hline 9 & Icterohaemorrhagiae & Icterohaemorrhagiae & Bianchi 1 & L. interrogans & Pathogenic \\
\hline 10 & Lora & Australis & Riccio 37 & L. interrogans & Pathogenic \\
\hline 11 & Pomona & Pomona & Mezzano I & L. interrogans & Pathogenic \\
\hline 12 & Saxkoebing & Sejroe & Mus 24 & L. interrogans & Pathogenic \\
\hline 13 & Zanoni & Pyrogenes & Zanoni & L. interrogans & Pathogenic \\
\hline 14 & Sejroe & Sejroe & Topo 1 & L. borgpetersenii & Pathogenic \\
\hline 15 & Mini & Mini & Sari & L. borgpetersenii & Pathogenic \\
\hline 16 & Castellonis & Ballum & Castellon 3 & L. borgpetersenii & Pathogenic \\
\hline 17 & Grippotyphosa & Grippotyphosa & Moskva V & L. kirschneri & Pathogenic \\
\hline 18 & Panama & Panama & CZ $214 \mathrm{~K}$ & L. noguchii & Pathogenic \\
\hline 19 & Hurstbridge & Hurstbridge & BUT6 & L. fainei & Intermediate \\
\hline 20 & Patoc & Semaranga & Patoc 1 & L. biflexa & Saprophytic \\
\hline
\end{tabular}

According to Brenner et al. [5].

Results of the pattern-matching process were expressed as identifying scores varying from 0 to 3.0 ; a score of $\geq 2.0$ indicates a species level identification; a score of 1.7-2.0 indicates a genus level identification and a score of $\leq 1.7$ is regarded as an unreliable identification.

\section{MALDI-TOF MS database and dendrogram}

MALDI-TOF MS analysis of reference Leptospira strains representative of 6 species (L. interrogans, L. kirschneri, L. borgpetersenii, L. biflexa, L. noguchii, and L. fainei) was performed.

A total of 40 replicates for each strain were performed and the obtained spectra were analysed with Flex Analysis software (Version 3.3). For each of the strains belonging to these 6 species, a MSP (Main Spectrum Profile) spectrum was obtained using the MALDI-Biotyper software (Bruker Daltonics, Germany) and then loaded in the Bruker Daltonics database (version 3.1.2.0). Subsequently, a blind trial was performed by using the 20 Leptospira strains included in the database.

Spectra obtained in this study were matched with the commercial Bruker database previously added by us with spectra from Brachyspira sp. and Borrelia sp. [22,23].

Cluster analysis was performed based on comparison of strain specific main spectra (MSP dendrogram) created as previously described [22].

\section{ClinProTools statistical analysis}

The same MALDI-TOF MS spectra previously selected for the database implementation were re-analysed with Flex Analysis software to perform the "smoothing" and the "baseline" for each spectrum. Subsequently, in order to compare the supplemented spectra and to identify specific discriminating peaks within the different analysed serovars, a statistical analysis was performed.

Then, the obtained spectra were loaded in an equal number in ClinProTools software version 2.2 (Bruker Daltonics) automatically recalibrated.

The analysis was performed within the range from 3,000 to $12,000 \mathrm{Da}$. To compare each strains the following specific algorithms of the software were used: Quick Classifier (QC) and Supervised Neural Network (SNN), that automatically give the highest value of "recognition capability (RC)" together with the highest value of "cross validation $(\mathrm{CV})$ " with the highest number of peaks (from 1 to 25), and the Genetic Algorithm that gives a value of $\mathrm{RC}$ together with a value of $\mathrm{CV}$ based on the maximum number of peaks selected by the operator. The classifications obtained with the 3 models were compared and the spectra considered to be different by at least 2 of the 3 models were recognized as "outliers" and eliminated. Moreover, in order to standardize the number of spectra analysed for each strain of each tested species, after the 
removal of the "outliers", 20 spectra were selected and then re-analysed by the same 3 statistical algorithms which displayed a list of discriminating peaks for the analysed spectra according to the selected algorithm. The algorithm with the highest score of $\mathrm{RC}$ and the highest value of $\mathrm{CV}$, taking into account also the number of the peaks utilized to obtain the model, was chosen to analyze the discriminating peaks that were displayed in a report. The presence or the absence of each discriminating peak was evaluated by comparing each average spectra automatically created from the replicates of each strain with the total average spectrum created with all the replicates loaded in the software.

\section{Results}

\section{MALDI-TOF MS database and dendrogram}

Leptospira protein profiles obtained in this study were found to be original, matching none of the existing profile in the database (score of the best match $<1.3$ ), when compared with the Bruker Daltonics database containing about 4,000 spectra corresponding to more than 2,000 bacterial species, including Brachyspira sp. and Borrelia sp. [22,23].

The spectra obtained by MALDI-TOF MS automatic acquisition were analysed by using Flex Analysis software. The replicates with an intensity $<10^{4}$ arbitrary units as well as those with a profile highly different from the others were discarded.

The spectra obtained by the analysis of the 20 reference strains tested were grouped into 6 main classes corresponding to the 6 species L. interrogans, L. borgpetersenii, L. kirschneri, L. biflexa, L. noguchii, and L. fainei (Figure 1), highlighting species-specific protein profiles with unique peaks in each species analysed.

The replicates, previously selected by Flex Analysis software, were used to create a MSP spectrum for each reference strain using MALDI-Biotyper software. The obtained MSP spectra were supplemented in our Bruker Daltonics database in order to identify Leptospira strains at the species level. These profiles proved to be reproducible in a second independent experiment performed after the database supplementation and a correct identification at the species and the serovar level was found for all replicates of each strain (score of the best match $>2.3$ ).

This reproducibility was observed with different lots of EMJH medium; moreover, each session was validated by external negative and positive controls: in all experiments the negative control spots yielded no peaks or faint profiles which were not identifiable by the system, and the positive control spots yielded $E$. coli identification with an identification score of 2-2.5.

Using the software MALDI Biotyper version 3.1 the 20 Leptospira protein reference spectra were visualized in a dendrogram (Figure 2). L. biflexa and L. fainei were located in a branch while L. interrogans, L. kirschneri,
L. noguchii and L. borgpetersenii clusterized in a separate one. The latter was further divided into two groups respectively containing, L. borgpetersenii on one side and L. interrogans, L. kirschneri, and L. noguchii on the other side.

\section{ClinProTools statistical analysis}

In order to visualize and identify the discriminatory peaks among the different serovars of L. interrogans and L. borgpetersenii analysed in this study, the spectra used for the MSP spectra creation were analysed by ClinProTools software and each replicates were shown on a two-dimensional plane; by default the first two best discriminating peaks of the current statistic sort order were displayed (Figure 3).

In order to identify specific peaks able to discriminate at serovar level, the spectra obtained for the species L. interrogans and L. borgpetersenii, respectively, were analysed by the statistical software.

The whole of the spectra of the strains within the same species were analysed applying different algorithms available in the software. For the species L. interrogans the SNN model showed a RC of $100 \%$ with a CV of $79.29 \%$ with 20 discriminating peaks that are reported in Table 2. In particular, the serovar Autumnalis was characterized by the presence of only 3 peaks $(3,684 \mathrm{Da}$, $5,527 \mathrm{Da}$, and $11,049 \mathrm{Da}$ ), the serovar Bratislava by the presence of all the peaks except for the peak of 5,671 Da and 6,915 Da. Analogously, for the remaining serovars the combinations of the presence or the absence of the discriminating peaks shown in Table 2 resulted to be discriminatory among serovars.

Discriminating peaks occurred also within the species L. borgpetersenii (Table 3). In particular, the presence of the peak of 3,024 Da was characteristic of the serovar Mini, the absence of the peak of 8,068 Da was characteristic of the serovar Sejroe.

\section{Discussion}

There is an urgent need for new diagnostic tools and typing methods easy to perform and readily available for public health and research laboratories for the important but neglected and understudied human disease that is leptospirosis: MALDI-TOF MS applied to the identification of leptospires described in this study enables simplicity, rapidity and cost-effectiveness that are unachievable with current technologies utilized for the typing of these bacteria. MALDI-TOF MS was already successfully applied to the identification of Leptospira sp. strains by Djelouadji et al. [7] and Rettinger et al. [24]. In this study, we made a further contribution to evaluate the usefulness of MALDI-TOF MS in the field of leptospirosis and we accomplished two objectives: i) to supplement our Bruker Daltonics database by using our own panel of leptospires 


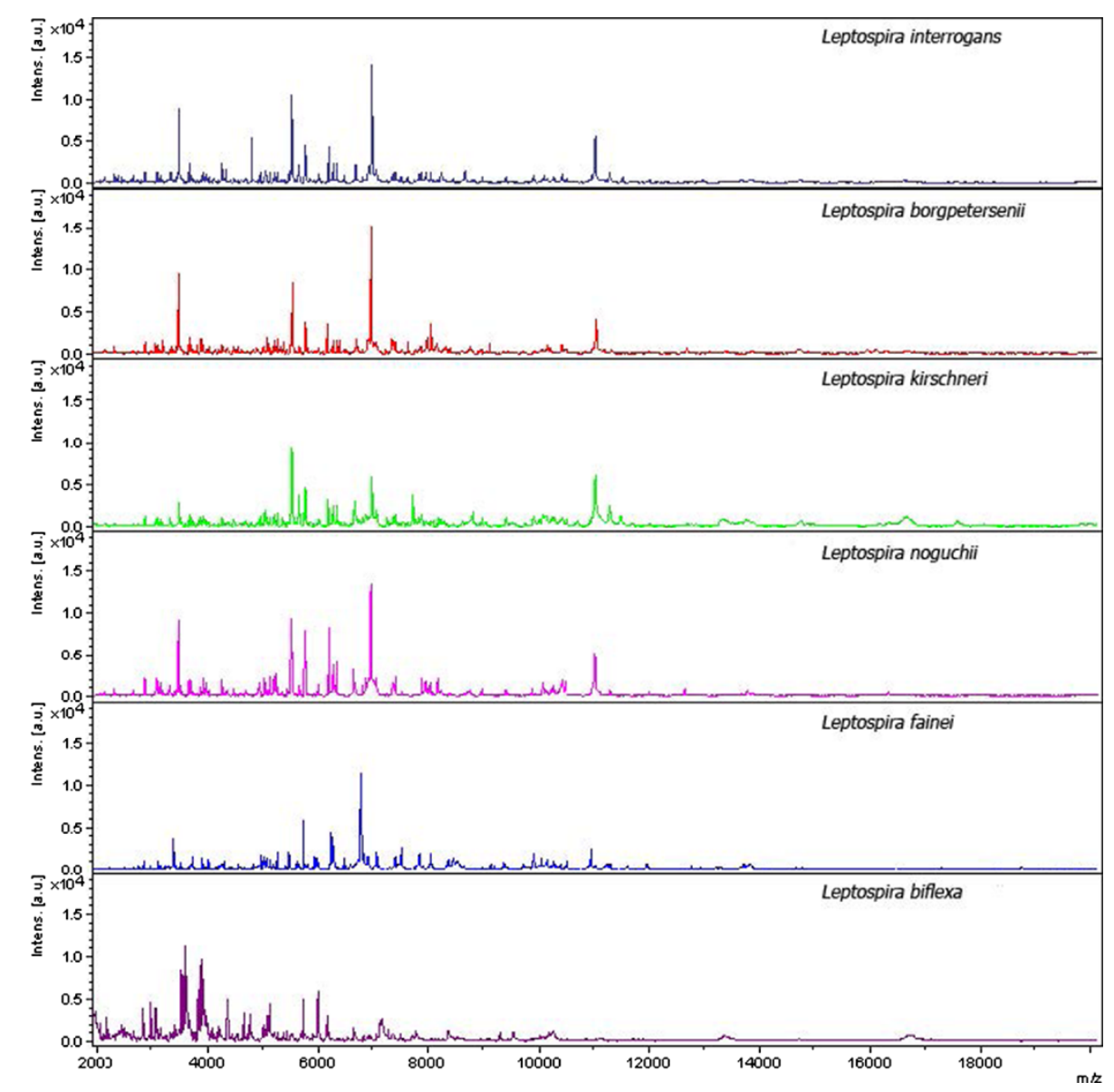

Figure 1 Spectra obtained by analysing reference strains of the 6 Leptospira species by MALDI-TOF MS.

including reference pathogenic and saprophytic strains circulating in Italy in order to assess the possible application of MALDI-TOF MS to the diagnosis of leptospirosis in our reference laboratory; ii) to create in our laboratory a statistical model able to differentiate at serovar level, by discriminating peaks, unknown strains belonging to L. interrogans and L. borgpetersenii species, respectively for epidemiological purposes.

As reported also by Rettinger et al. [24], by using a very simple protocol we obtained an interpretable identifying protein profile for each of the 20 analysed strains starting from a minimal concentration of $1 \times 10^{6}$ organisms/ $\mathrm{ml}$ that is achieved with no special requirements of growth conditions: this indicates the possibility of using early Leptospira cultures to perform MALDI-TOF MS consequently reducing the delay for their identification. Moreover, we further observed that profiles were robust, being independent from the EMJH lot used for cultivation.

In this study we were able to group by Flex Analysis software the spectra obtained by MALDI-TOF MS of the 20 reference strains analysed into 4 main classes corresponding to the 6 Leptospira sp. showing species-specific protein profiles with unique peaks in each species analysed. Moreover, it was also possible to observe little differences among the profiles of the analysed strains within a given species (namely $L$. interrogans and $L$. borgpetersenii, for which more than one strain was analysed), probably due to the presence of differences among the protein patterns of distinct serovars, as subsequently confirmed using ClinProTools analysis that showed serovar-specific peaks. This observation is related to the strains overall analysed in this study taking into account that the ClinProTools software results are specific for the panel of the strains analysed in each experimental session. For this reason confirming the result obtained by Rettinger et al. [24], we cannot definitely conclude that we identified universal serovar-specific peaks, since we used a selected panel of serovars in this study. Nevertheless, a serovar included in the reference panel of the species analysed could be likely assigned without genetic analysis.

The dendrogram obtained by Maldi Biotyper analysis on the basis of the protein patterns of the six species tested reflects the phylogenetic tree based on 16SrRNA sequencing reported in the literature [24] showing Leptospira 


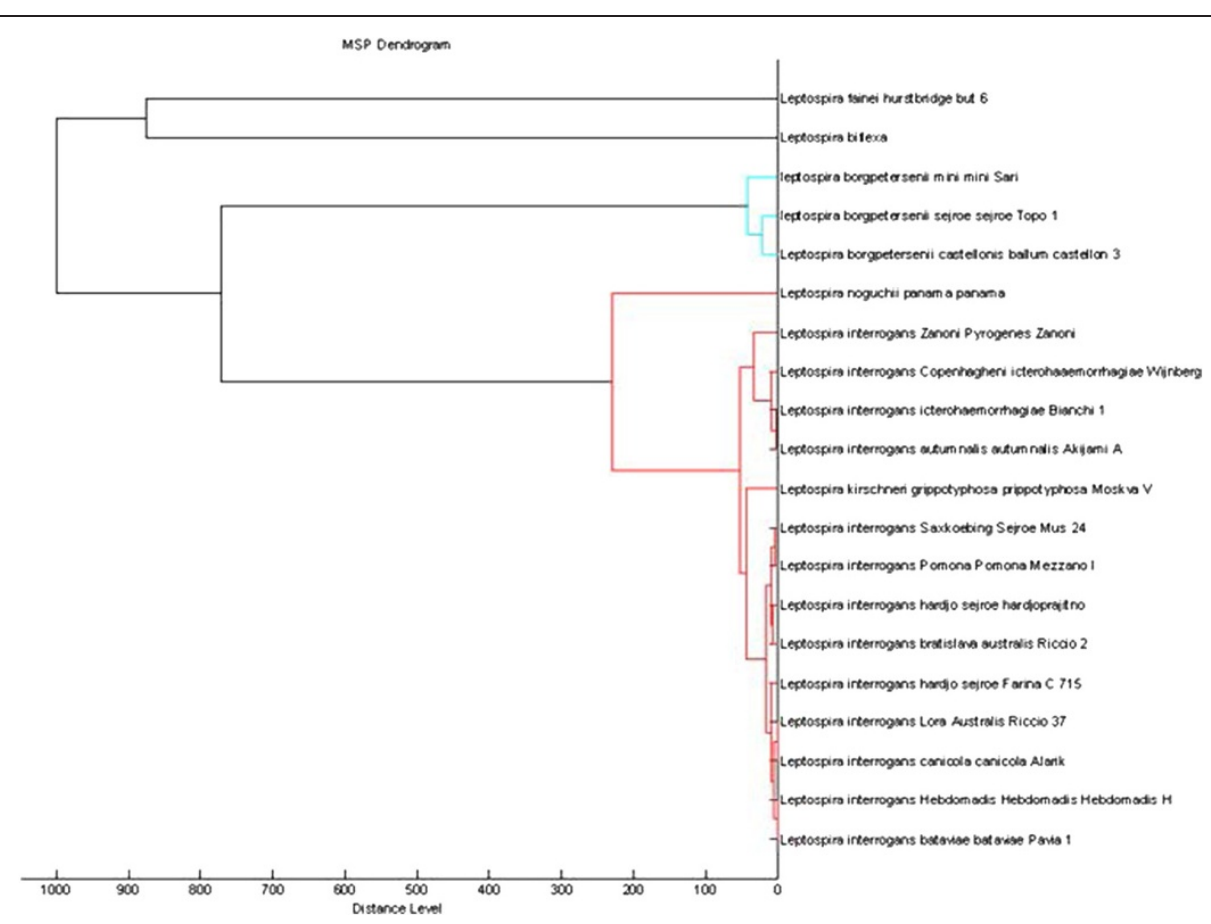

Figure 2 "Main Spectra Profiles"-based dendrogram of the 20 Leptospira sp. reference strains analysed in this study.

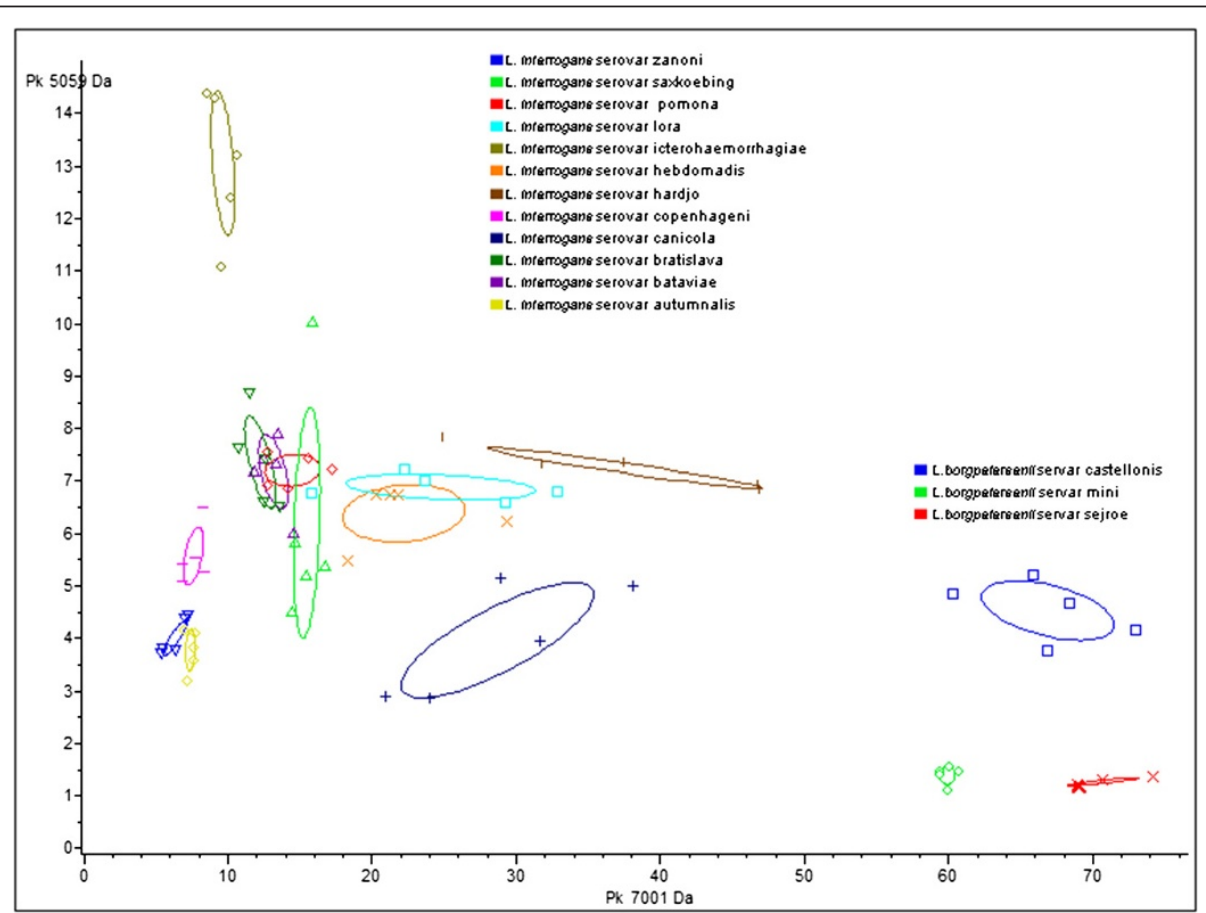

Figure 3 Differentiation of $L$. interrogans and $L$. borgpetersenii serovars by ClinProTools software. "2D Peak Distribution View" displaying the distribution of the different 15 serovars (by way of example, 5 replicates per serovar are shown) analysed on the basis of the first two best discriminating peaks among the spectra of all serovars considered. The symbols represent the ratio area/intensity with respect to the two peaks considered for each spectrum, the same symbols represent replicates of the same serovar and the ellipses represent the standard deviation within the same serovar. 
Table 2 Differentiating peaks obtained by statistical analysis of 12 serovars belonging to the species $L$. interrogans

Serovars $\quad$ Peak mass ( $\mathrm{m} / \mathrm{z})$ representing the protein size in Dalton

\begin{tabular}{|c|c|c|c|c|c|c|c|c|c|c|c|c|c|c|c|c|c|c|c|c|}
\hline \multirow[t]{2}{*}{ Serovars } & \multicolumn{20}{|c|}{$\operatorname{ein} \mathrm{L}$} \\
\hline & 3,506 & 3,684 & 3,952 & 5,059 & 5,527 & 5,658 & 5,671 & 5,782 & 6,193 & 6,284 & 6,356 & 6,915 & 6,941 & 6,970 & 7,013 & 7,059 & 7,077 & 7,902 & 11,049 & 11,311 \\
\hline Autumnalis & - & + & - & - & + & - & - & - & - & - & - & - & - & - & - & - & - & - & + & - \\
\hline Canicola & $\S$ & - & $\S$ & - & - & $\S$ & - & $\S$ & + & $\S$ & - & + & + & + & + & + & + & $\S$ & - & $\S$ \\
\hline Icterohaemorrhagiae & $\S$ & + & + & + & + & $\S$ & - & + & - & - & + & - & - & $\S$ & + & $\S$ & + & + & + & + \\
\hline Hardjo & + & - & + & + & - & - & + & + & + & + & + & + & + & + & + & + & + & $\S$ & - & $\S$ \\
\hline Hebdomadis & $\S$ & + & + & + & - & - & - & $\S$ & + & - & - & - & $\S$ & $\S$ & - & + & + & $\S$ & - & - \\
\hline Pomona & + & + & + & + & - & - & - & + & + & + & + & - & $\S$ & + & + & + & + & + & - & - \\
\hline Saxkoebing & - & - & $\S$ & + & - & - & - & $\S$ & - & $\S$ & $\S$ & - & $\S$ & + & + & + & + & + & + & $\S$ \\
\hline Batavie & + & + & $\S$ & + & + & + & + & - & + & - & - & + & + & + & + & + & + & $\S$ & + & + \\
\hline Bratislava & + & + & + & + & + & + & $\S$ & + & + & + & + & $\S$ & + & + & + & + & + & + & + & + \\
\hline Lora & - & - & + & $\S$ & - & - & + & + & $\S$ & + & + & - & - & - & - & $\S$ & $\S$ & + & - & - \\
\hline Copenhageni & - & + & + & $\S$ & + & - & - & - & + & + & - & - & - & - & - & - & $\S$ & $\S$ & + & - \\
\hline Zanoni & - & + & - & - & + & + & + & - & - & - & - & + & + & - & - & - & - & - & $\S$ & + \\
\hline
\end{tabular}

The identification of each serovar is derived from the combinations of the 20 discriminating considered peaks.

+: peak with area/intensity of the Serovar Average Spectrum higher than the Total Average Spectrum for the respective considered peak.

$\because$ no peak found.

§: peak with area/intensity of the Serovar Average Spectrum lower than the Total Average Spectrum for the respective considered peak. 
Table 3 Differentiating peaks obtained by statistical analysis of 3 serovars belonging to the species L. borgpetersenii

\begin{tabular}{|c|c|c|c|c|c|}
\hline \multirow[t]{2}{*}{ Serovars } & \multicolumn{5}{|c|}{ Peak mass $(\mathrm{m} / \mathrm{z})$ representing the protein size in Dalton } \\
\hline & 3,024 & 3,499 & 6,728 & 7,357 & 8,068 \\
\hline Castellonis & - & + & $\S$ & + & + \\
\hline Mini & + & $\S$ & + & - & + \\
\hline Sejroe & - & + & + & + & - \\
\hline
\end{tabular}

The identification of each serovar is derived from the combinations of the 5 discriminating considered peaks.

+: peak with area/intensity of the Serovar Average Spectrum higher than the Total Average Spectrum for the respective considered peak.

- no peak found.

§: peak with area/intensity of the Serovar Average Spectrum lower than the

Total Average Spectrum for the respective considered peak.

species clustered according to their pathogenicity (pathogenic and saprophytic strains clearly separated into different clusters) and confirming the comparability of the results obtained by mass spectrometry and by molecular typing methods.

Leptospira identification has been traditionally accomplished by serological methods [8] which do not always give unambiguous results. Further, the adoption of a genotypic classification complicated the identification of leptospires because of the same serovars may be found in more than one species and some species contain both pathogenic and non pathogenic serovars [5,7], thus the determination of serovar is no longer sufficient to assign an isolate to its correct species. Actually, for public health purposes it has become essential to identify not only the serovar but also the species of isolates in order to accurately track the transmission of Leptospira during outbreaks.

MALDI-TOF MS shows, in the laboratory diagnosis of leptospirosis, as previously reported for other bacterial diseases, several advantages over currently available methods of species-level identification [21,22]. First of all, the minimal sample preparation and simple acquisition combined with its potential for high throughput sample automation make the technique a valuable and rapid identification method (it takes only one hour and thirty minutes to obtain the results) [7,25]. Finally, the method is more cost-effective, with an estimated total cost of 0.50 Euro per strain in our experience. All these considerations put this methodology in a central position in microbiology also in the case of such a neglected pathogen as Leptospira.

Our results obtained with 20 reference Leptospira strains showed that MALDI-TOF MS followed by the analysis with ClinProTools software could be used as a first-line technique for rapid, cheap and reliable identification of Leptospira strains at the species level and possibly to discriminate certain serovars that belong to the same genomospecies, as previously proposed by Rettinger et al. [24]. The MALDI-TOF MS spectra available at the moment will serve as a basis to create a database for further identification of unknown strains at serovar level, and our data strongly suggest that such an application could be likely successful.

In recent years, the development of genetic tools and the availability of complete genome sequences of pathogenic Leptospira have made it possible to apply state-ofthe-art approaches to determine the virulence mechanisms of leptospires [2]; likewise, MALDI-TOF MS could prove in the future a useful tool to evidence the existence of different biotypes showing different protein profiles, possibly related to different pathogenic virulence factors, to be further investigated by more sophisticated approaches in order to gain an insight into the biology of leptospires.

\section{Conclusions}

MALDI-TOF MS emerged over the last years as a useful technology to be applied in the clinical microbiology setting and it has revolutionized the work-flow also in our laboratory, enabling rapid identification of bacteria and yeasts for clinical diagnosis.

In this study, we confirmed that MALDI-TOF MS could be a powerful method for research and diagnostic in the field of leptospirosis with broad applications ranging from the detection and identification of pathogenic leptospires for diagnostic purposes to the typing of pathogenic and non-pathogenic leptospires for epidemiological purposes in order to enrich our knowledge about the epidemiology of the infection in different areas, taking into account that the typing of the circulating leptospiral strains is the first step towards identifying reservoirs and generating control strategies.

\section{Competing interests}

The authors declare the absence of any competing interests.

\section{Authors' contribution}

Designed the experiments: AC, GP, CG. Performed the experiments: GP, CG, SM, MB, MP, SR. Analysed the data: AC, FDC, MCA, CC, MCM. Contributed reagents, materials, analysis tools: $A C$. Wrote the manuscript: $A C, G P, C G, S M$, MB. Conceived and supervised the project: AC, CC. All authors read and approved the final manuscript.

\section{Acknowledgements}

This study was supported by the Ministry of University and Scientific Research Grant FIL, Parma, Italy.

We thank Dr. Mathieu Picardeau, French National Reference Centre for Leptospirosis, Pasteur Institute, Paris, France, for providing us with the strains L. fainei and L. noguchii.

We thank Dr. Marco Fagioni for technical assistance.

Received: 17 May 2014 Accepted: 21 May 2014

Published: 2 June 2014

\section{References}

1. Bharti AR, Nally JE, Ricaldi JN, Matthias MA, Diaz MM, Lovett MA, Levett PN, Gilman RH, Willig MR, Gotuzzo E, Vinetz JM, Peru-United States Leptospirosis Consortium: Leptospirosis: a zoonotic disease of global importance. Lancet Infect Dis 2003, 3:757-771. 
2. Ko Al, Goarant C, Picardeau M: Leptospira: the dawn of the molecular genetics era for an emerging zoonotic pathogen. Nat Rev Microbiol 2009, 7:736-747.

3. Levett PN: Leptospirosis. Clin Microbiol Rev 2001, 4:296-326.

4. Adler B, Lo M, Seemann T, Murray GL: Pathogenesis of leptospiroses: the influence of genomics. Vet Microbiol 2011, 153:73-81.

5. Brenner D, Kaufmann AF, Sulzer KR, Steigerwalt AG, Rogers FC, Weyant RS: Further determination of DNA relatedness between serogroups and serovars in the family Leptospiraceae with a proposal for Leptospira alexanderi sp. nov. and four new Leptospira genomospecies. Int I Syst Bacteriol 1999, 49:839-858.

6. Cerqueira GM, Picardeau M: A century of Leptospira strain typing. Infect Genet Evol 2009, 9:760-768

7. Djelouadji Z, Roux V, Raoult D, Kodjo A, Drancourt M: Rapid MALDI-TOF mass spectrometry identification of Leptospira organisms. Vet Microbiol 2012, 158:142-146.

8. Postic D, Merien F, Perolat P, Baranton G: Laboratory methods - Biological diagnosis for Leptospirosis. In Biological diagnosis Leptospirosis - Lyme borreliosis. 2nd enlarged edition. Paris: Institut Pasteur; 2000:139-196.

9. Perolat P, Chappel RJ, Adler B, Baranton G, Bulach DM, Billinghurst ML, Letocart M, Merien F, Serrano MS: Leptospira fainei sp. nov., isolated from pigs in Australia. Int J Syst Bacteriol 1998, 48:851-858.

10. Ciceroni L, Ciarrocchi S, Ciervo A, Petrucca A, Pinto A, Calderaro A, Viani I, Galati L, Dettori G, Chezzi C: Differentiation of leptospires of the serogroup Pomona by monoclonal antibodies, pulsed-field gel electrophoresis and arbitrarily primed polymerase chain reaction. Res Microbiol 2002, 153:37-44.

11. Herrmann JL, Bellenger E, Perolat P, Baranton G, Saint Girons I: Pulsed-field gel electrophoresis of Notl digests of leptospiral DNA: a new rapid method of serovar identification. J Clin Microbiol 1992, 30:1696-1702.

12. Zuerner RL, Ellis WA, Bolin CA, Montgomery JM: Restriction fragment length polymorphisms distinguish Leptospira borgpetersenii serovar hardjo type hardjo-bovis isolates from different geographical locations. J Clin Microbiol 1993, 31:578-583.

13. Majed Z, Bellenger E, Postic D, Pourcel C, Baranton G, Picardeau M: Identification of variable-number tandem-repeat loci in Leptospira interrogans sensu stricto. J Clin Microbiol 2005, 43:539-545.

14. Ahmed N, Devi SM, Valverde M, Vijayachari P, Machang'u RS, Ellis WA, Hartskeerl RA: Multilocus sequence typing method for identification and genotypic classification of pathogenic Leptospira species. Ann Clin Microbiol Antimicrob 2006, 5:28.

15. Branger C, Blanchard B, Fillonneau C, Suard I, Aviat F, Chevallier B, André-Fontaine G: Polymerase chain reaction assay specific for pathogenic Leptospira based on the gene hap1 encoding the hemolysis-associated protein-1. FEMS Microbiol Lett 2005, 243:437-445.

16. La Scola B, Bui LT, Baranton G, Khamis A, Raoult D: Partial rpoB gene sequencing for identification of Leptospira species. FEMS Microbiol Lett 2006, 263:142-147.

17. Merien F, Amouriaux P, Perolat P, Baranton G, Saint Girons I: Polymerase chain reaction for detection of Leptospira sp. in clinical samples. J Clin Microbiol 1992, 30:2219-2224

18. Natarajaseenivasan K, Vijayachari P, Sharma S, Sugunan AP, Vedhagiri K, Selvin J, Sehgal SC: FlaB PCR-based identification of pathogenic leptospiral isolates. J Microbiol Immunol Infect 2010, 43:62-69.

19. Slack AT, Symonds ML, Dohnt MF, Smythe LD: Identification of pathogenic Leptospira species by conventional or real-time PCR and sequencing of the DNA gyrase subunit B encoding gene. BMC Microbio/ 2006, 6:95.

20. El-Khéchine A, Couderc C, Flaudrops C, Raoult D, Drancourt M: Matrix-assisted laser desorption/ionization time-of-flight mass spectrometry identification of mycobacteria in routine clinical practice. PLoS One 2011, 6:e24720.

21. Seng P, Drancourt M, Gouriet F, La Scola B, Fournier PE, Rolain JM, Raoult D: Ongoing revolution in bacteriology: routine identification of bacteria by matrix-assisted laser desorption ionization time-of-flight mass spectrometry. Clin Infect Dis 2009, 15:543-551.

22. Calderaro A, Piccolo G, Montecchini S, Buttrini M, Gorrini C, Rossi S, Arcangeletti MC, De Conto F, Medici MC, Chezzi C: MALDI-TOF MS analysis of human and animal Brachyspira species and benefits of database extension. J Prot 2013, 78:273-280

23. Calderaro A, Gorrini C, Piccolo G, Montecchini S, Buttrini M, Rossi S, Piergianni M, Arcangeletti MC, De Conto F, Chezzi C, Medici MC
Identification of Borrelia species after creation of an in-house MALDI-TOF MS database. PLoS One 2014, 12(2):e88895.

24. Rettinger A, Krupka I, Grünwald K, Dyachenko V, Fingerle V, Konrad R, Raschel H, Busch U, Sing A, Straubinger RK, Huber I: Leptospira sp. strain identification by MALDI TOF MS is an equivalent tool to 16S rRNA gene sequencing and multi locus sequence typing (MLST). BMC Microbiol 2012, 12:185.

25. Ayyadurai S, Flaudrops C, Raoult D, Drancourt M: Rapid identification and typing of Yersinia pestis and other Yersinia species by matrix-assisted laser desorption/ionization time-of-flight (MALDITOF) mass spectrometry. BMC Microbiol 2010, 10:285.

doi:10.1186/1756-0500-7-330

Cite this article as: Calderaro et al.: Leptospira species and serovars identified by MALDI-TOF mass spectrometry after database implementation. BMC Research Notes 2014 7:330.

\section{Submit your next manuscript to BioMed Central and take full advantage of:}

- Convenient online submission

- Thorough peer review

- No space constraints or color figure charges

- Immediate publication on acceptance

- Inclusion in PubMed, CAS, Scopus and Google Scholar

- Research which is freely available for redistribution 